\title{
AN UNUSUAL COMPLICATION OF TRAUMATIC CATARACT
}

\author{
BY \\ T. W. LETCHWORTH \\ LONDON
}

A FOREIGN body entering an eye, without the patient being aware of it, is an accident that is not uncommon.

In the case reported here a piece of steel was present in the lens unsuspected by the patient ; but an additional feature of considerable interest is the fact that the lens matter, which had become opaque as a result of the injury, suddenly became extruded into the vitreous.

History.-A man, aged 44 years, came to the Royal Eye Hospital complaining that he had noticed the sight of the right eye misty for three weeks. There was no recollection of any accident or injury to the eye.

Examination. Right eye.-Vision was reduced to hand movements, projection good, pupil reactions and tension normal.

The cornea and iris were normal, but the anterior chamber was rather shallow. No clear red reflex could be obtained, owing to extensive lens opacities irregularly distributed, but most dense towards the centre. Lying embedded in the substance of the lens, towards the temporal side, could be seen a glistening foreign body metallic in nature.

The shallowness of the anterior chamber was due to swelling of the lens. The eye was white with no suggestion of irritation, or any precipitate on the cornea.

Left eye.-Vision was full and the eye normal in every respect.

Operative interference was deemed inadvisable at this stage. It was thought better to wait until the lens should become completely opaque, and then remove the lens and with it the foreign body. The man was therefore kept under observation as an out-patient.

Subsequent history.- Seen a week later, the lens was much more opaque and swollen, and, in consequence, the anterior chamber more shallow, but the eye was not irritable.

A week later still the lens was almost completely opaque, the anterior chamber still more shallow, and the tension distinctly raised. A preliminary iridectomy was decided upon and done seven days later. Neither the operation nor the convalescence presented any unusual feature. The patient was seen once a week for the subsequent three weeks, and on every occasion the eye was white and quiet, and by then the lens was ready for extraction.

Just a month after the operation the patient presented himself because of a sudden sharp pain he had experienced in the right eye four days before. 
Examination revealed a deep anterior chamber, with slight rise of tension and the opaque lens could be seen lying in the vitreous chamber below and behind the pupil. It was, therefore, necessary to attempt to remove it with the foreign body.

Operation.-The operation was carried out three days later, that is seven days after the experience of pain reported by the patient.

As the vitreous would in all probability present in the wound it was thought advisable to have a conjunctival flap prepared to cover the wound completely at the end of the operation. This was done by picking up a fold of conjunctiva at " 10.30 o'clock" $1 \mathrm{~cm}$. from the limbus, carrying the incision radially to the limbus and then detaching the conjunctiva from the cornea all the way round above to " 3 o'clock." Above this incision a flap of conjunctiva was raised up so that it could be drawn down over the area of the incision and fixed by one stitch from the point of this flap to the ocular conjunctiva on the lower and temporal part of the eye.

The stitch was then inserted to be tightened when the operation was complete. The ordinary cataract section was then made. No vitreous presented. A vectis was introduced but before it could enter the vitreous cavity a definite resistance had to be overcome, due probably to the intact anterior capsule of the lens.

The lens was considerably broken up and the foreign body was removed with the largest mass of lens matter, several smaller parts having to be removed afterwards. Practically all the lens matter was removed with little loss of vitreous.

The stitch was then tightened bringing the conjunctival flap over the wound.

The foreign body proved to be a bit of steel $2 \mathrm{~mm}$. in length and weighing $7 \mathrm{mgrs}$. The eye is progressing favourably.

Comments.-The lens matter, apparently, escaped into the vitreous by bursting through the posterior capsule, the anterior capsule remaining in its place and offering resistance to the vectis.

It is impossible to say how long the foreign body had been in the lens, but owing to the rapidity with which the lens swelled up after it was first seen it cannot have been long before the mistiness of sight was first noticed. Whether the swollen lens burst through an intact posterior capsule or enlarged a pre-existing wound is difficult to decide, but the absence of evidence of the steel having entered through the cornea lends support to the view that the foreign body entered the lens through the posterior capsule. In any case this complication of opaque and swollen lens matter becoming extruded into the vitreous is worth bearing in mind in cases of traumatic cataract in which the lens shows rapid swelling. In such cases a waiting policy would appear not to be devoid of danger. 